\title{
Improved Identification and Assessment of Heat-Related Illnesses in Pinal County, AZ
}

\section{Dametreea Carr}

Infectious Disease and Epidemiology Section, Pinal County Public Health Services District, Florence, Arizona, United States

Objective

Determine risk factors and risk populations for heat-related illnesses in Pinal County, AZ by improving HRI case identification and assessment.

\section{Introduction}

Extreme heat and related illnesses are a critical concern in Arizona from May to September each year. From 2008 to 2016, Arizona medical facilities had an average of 1,790 emergency visits and 442 hospital admissions for heat-related illnesses (HRI) during the summer months. In 2016 alone, Arizona Emergency Departments (EDs) received a total of 2,484 visits for HRI and 527 of these cases were admitted as inpatients [1]. Pinal County, which has an estimated population of 430,237 individuals [2], contributes to the number of HRI visits to Arizona Emergency Departments that occur each year. In order to determine the burden of HRIs within the county, Pinal County Public Health Services District (PCPHSD) began to conduct heat-related illness surveillance in 2017 and found that 149 HRI cases had been reported that year[3]. PCPHSD continued to conduct HRI surveillance through summer 2018 to build on surveillance activities from 2017 and meet the following goals:

- Improve HRI case identification and assessment through enhanced HRI surveillance and interview processes,

- Determine risk factors and risk populations for HRIs in Pinal County, and

- Recommend and implement practical interventions to prevent HRIs among Pinal residents.

\section{Methods}

The process to improve identification and assessment of HRI cases among Pinal residents included the use of the National Syndromic Surveillance Program's (NSSP) ESSENCE for case identification, and Qualtrics survey software to collect additional information from confirmed cases regarding their heat exposure.

First, cases were identified using ESSENCE syndromic surveillance system, which identifies cases based on specific queries and definitions. PCPHSD used the definition for Heat Illness version 1 to identify cases who's Chief Complaint (CC) and/or hospital Discharge Diagnosis (DD) included key HRI terms. Next, PCPHSD utilized two ESSENCE queries to search cases by "patient location" and "facility location." These two queries were used to ensure that all HRI cases among Pinal County residents were identified regardless of case address or the facility visited. The second step in the process included de-duplication of cases identified in each query and adding them to a line list for further assessment. PCPHSD then conducted a thorough medical chart review for each case to determine if they met the HRI case definition. Confirmed cases met the following criteria:

- Identification in ESSENCE as an HRI,

- Medical records compatible with HRI clinical symptoms,

- Symptom onset within 24 hours of heat exposure,

- HRI visit to an ED between May $1^{\text {st }}$ and September $30^{\text {th }}, 2018$, and

- Resident of Pinal County.

- Exclusion: heat exposure not related to environmental/natural heat.

Step three of the process included the conduction of HRI case interviews using Qualtrics survey software. Interview questions were designed to obtain information not included in case medical records, and to collect information regarding risk factors, risk populations, and potential areas of intervention. Interviews were completed within 7-10 minutes, included a maximum of three call attempts to each case, and included a follow-up text message. Cases were sent an information packet if they expressed interest in receiving additional information at the conclusion of their interview. The information packet included a thank you letter, heat safety tips, local resources for homelessness and utility assistance, and heat relief options available through the Pinal County Heat Relief Network. The final step of the process included data analysis to determine areas and modes of intervention to prevent HRIs among Pinal residents.

SDS Annual Conference Proceedings 2019. This is an Open Access article distributed under the terms of the Creative Commons AttributionNoncommercial 4.0 Unported License (http://creativecommons.org/licenses/by-nc/3.0/), permitting all non-commercial use, distribution, and reproduction in any medium, provided the original work is properly cited. 


\section{Results}

ESSENCE identified 168 HRI cases from May $1^{\text {st }}$ to August $31^{\text {st }}, 2018$. Of these cases, 103 were confirmed. (Note that 48 cases from August and two from July are still under chart review). Preliminary findings for confirmed cases from May $1^{\text {st }}$ to July $31^{\text {st }}$ show that the majority of HRI cases in Pinal County are Non- Hispanic White (61.2\%), male (75.7\%), and range in age from 2059 years $(71.8 \%)$. Nearly one-third of reported HRI cases occurred among Casa Grande Residents (33 cases). This was followed by San Tan Valley with 20 cases, and Apache Junction with 16 cases. With regard to hospital visits, $86.4 \%$ of cases visited an ED within Pinal County. Other county ED visits were to Maricopa (10.7\%), Pima (1.9\%), and Coconino (1\%). The most frequently visited hospitals within Pinal County were Banner Casa Grande Medical Center $(50.5 \%)$ and Banner Goldfield Medical Center $(12.6 \%)$.

Assessment of cases by month shows that July had the greatest number of cases overall (51 cases) and the greatest number of cases in one day ( 5 cases). This was followed by the month of June with 41 cases total and a maximum of 3 cases in one day. Maximum temperatures in June and July reached $111^{\circ} \mathrm{F}$ and $116^{\circ} \mathrm{F}$, respectively.

With regard to response rate, $53.2 \%$ of confirmed cases completed an interview. Interview data are currently being assessed to determine risk factors and risk populations for HRI. Final results will include May to September HRI data, an analysis of risk factors (e.g. occupation, recreation, homelessness, access to heat- safety resources), an updated response rate, and the percentage of cases who requested an information packet. Final data analyses will be completed by December 2018.

\section{Conclusions}

Improvements to the process for HRI surveillance and case interviews has helped PCPHSD to more effectively identify and assess HRI cases. Utilizing two ESSENCE queries assisted with HRI case identification, and improvements to the case contact protocol and interview questionnaire increased response rate from $25 \%$ in 2017 [3] to 53.2\% in 2018. Other improvements included the use of follow-up text messaging and Spanish text/interview options. Preliminary results indicate that non-Hispanic white males between the ages of 20-59 years have a greater risk of getting an HRI than other demographic groups. Interventions should therefore be geared toward this population. Also, with HRIs occurring in multiple cities throughout Pinal County, it is recommended that the Pinal County Heat Relief Network expand outreach activities to recruit additional organizations to participate as heat relief stations.

Final analysis of interview data will take place to determine if HRIs among Pinal residents are related to occupation, recreation, socioeconomic status, or a combination of these. PCPHSD will use final results to provide targeted heat-safety information and resources to the most appropriate groups. It is the hope of PCPHSD that this project will benefit at-risk Pinal County residents and reduce the burden of HRIs during future summers.

\section{Acknowledgement}

Special thanks to Clancey Hill and Rachel Zenuk from Pinal County Public Health Services District; and Krystal Collier, Matthew Roach, Sara Chronister, Sara Johnston, Melissa Kretchmer, and Laura Erhart from Arizona Department of Health Services.

\section{References}

1. Centers for Disease Control and Prevention. Environmental Public Health Tracking Network, State Emergency Department Visits data. [Internet]. 2018. Available from: www.cdc.gov/ephtracking. Accessed on 10/12/2018.

2. United States Census Bureau. Quick Facts: Pinal County, AZ. [Internet]. 2017 Jul 1. Available from: https://www.census.gov/quickfacts/fact/table/pinalcountyarizona/PST045217\#viewtop.

3. Kent DC, Garcia RZ, Packard S, Briggs G, Hill C, et al. 2018. Enhanced Surveillance of heat-related illness in Pinal County. Online J Public Health Inform. 10(1), e111. doi: 10.5210/ojphi.v10i1.8829 